\title{
Comparative CFD Simulations of Gas Transport in Slug Flow from Periodic Arrays with Single or Multiple Bubbles
}

\author{
G. P. Oliveira ${ }^{a, 1}$, N. Mangiavacchi ${ }^{a}$, G. Anjos $^{a}$, \\ J. Pontes ${ }^{a}$, J. R. Thome ${ }^{b}$ \\ ${ }^{a}$ Group of Environmental Studies in Water Reservoirs - GESAR, \\ State University of Rio de Janeiro, Rio de Janeiro, Brazil \\ ${ }^{b}$ Heat and Mass Transfer Laboratory - LTCM, École Polytechnique Fédérale de Lausanne, \\ Lausanne, Switzerland \\ Received on January 01, 2015 / Accepted on March 15, 2015
}

\begin{abstract}
This paper presents a comparative study of transport effects in slug flows through numerical models established upon volume-preserving periodic arrays of bubbles. The behaviour of the flow is investigated under an adiabatic laminar regime as usually found in microchannels, as well as in microscale fluid applications. Full 3D Direct Numerical Simulations (DNS) are performed for three different cases of setups containing both single and multiple bubbles. Profiles of the bubbles' centroidal velocity are compared, as with the average mass flow rates calculated over the periodic boundaries of the domain.
\end{abstract}

Keywords: Finite element, slug flow, periodic boundary conditions, bubble.

\section{Introduction}

Slug flow (or Taylor flow) is a two-phase flow regime recognizable by capsule-shaped elongated bubbles that separate portions of liquid forming a scenario of high void fraction and large surface-areato-volume ratio [1], [2]. The mutual and quasiperiodic interaction among the bubbles occurring in this regime impacts directly mass transfer processes taking place mainly in gas-liquid flows. Consequently, several applications of slug flows have been developed in fields such as nuclear, biological and chemical engineering [3], [4], [5].

In the leading edge of the recent technologies applied to efficient cooling of microdevices, slug flows come up mainly due to the improved heat transfer properties reached in this regime. Such quest for operative mechanisms of high performance in heat dissipation uplifts interests to understand the dynamics

\footnotetext{
${ }^{1}$ E-mail Corresponding Author: gustavo.oliveira@uerj.br
}

of multiple bubbles inside microchannels. For this reason, different sights regarding both numerical and experimental methods are in promising development [6], [7], [8]. The study of elongated bubbles and their heat transfer effects were investigated numerically in recent papers [9] [10], reporting the necessity of simulations containing several bubbles.

The present paper focuses on an adiabatic lowReynolds modelling to study the gas transport effects of the slug flow from distinctive configurations of periodic arrays of bubbles conserving the same void fraction. Finite element techniques are implemented via an Arbitrary Lagrangian-Eulerian [11], [12] description coupled with periodic boundary conditions and pressure-driven flow as numerical strategies to represent the hydrodynamics of bubble trains occurring inside a microchannel. 


\section{Modelling of Bubbles in Periodic Arrays}

To represent mathematically the problems studied here, the model begins from a primal base configuration of periodic array $\mathcal{A}_{1}$ which is formed by a cylindrical domain of diameter $D$, length of period $L$ and periodic boundaries $\Gamma_{L}, \Gamma_{R}$ encircling a Taylor bubble of volume $V_{g}$ represented by a convex region $\Omega_{1}$. Operating as a branching process, two more configurations of arrays $\mathcal{A}_{2}$ and $\mathcal{A}_{3}$ are obtained by sectioning the longest bubble into two or three bubbles as depicted in Fig. 1. The length of the periodic cell is defined as a function of the diameter and preserves the total volume regardless of the configuration studied, as with the regions $\Omega_{2}$ and $\Omega_{3}$ that also keep the fractioned volume.



Figure 1: Diagram displaying the branch of a volume-preserving model into three periodic arrays of Taylor bubbles in a cartesian system.

Moreover, a moving frame technique is applied in the models by which the bubbles are kept fixed, while the cylindrical wall moves backwards with velocity equal to the average velocity of the bubbles' centroids. Furthermore, a negative pressure gradient is applied on the streamwise direction of the flow given by the positive $x$-axis so producing motion from the left side to the right one. Clearly, the average centroidal velocity is reduced to the centroidal velocity only for the case of a unique bubble.
The two-phase system aforementioned is governed by the dimensionless ALE/FE incompressible NavierStokes equations embedded with additional terms to take the contributions of the periodic pressure and interface force into account, being written, subsequently as

$$
\begin{aligned}
\frac{D(\rho \mathbf{u})}{D t}= & \beta \hat{\mathbf{i}}-\nabla P+\frac{1}{R e} \nabla \cdot\left[\mu\left(\nabla \mathbf{u}+\nabla \mathbf{u}^{T}\right)\right]+ \\
& \frac{1}{W e} \mathbf{f}+\frac{1}{F r^{2}} \rho \mathbf{g} \\
\nabla \cdot \mathbf{u}= & 0
\end{aligned}
$$

with

$$
\frac{D(\rho \mathbf{u})}{D t}=\frac{\partial(\rho \mathbf{u})}{\partial t}+(\mathbf{u}-\hat{\mathbf{u}}) \cdot \nabla(\rho \mathbf{u}),
$$

where $\mathbf{u}$ is the velocity field, $\rho, \mu$, are the reference density and viscosity of the fluid, respectively, $\hat{\mathbf{u}}$ is the mesh velocity [13], $\mathrm{g}$ the vector of standard constant gravity $g$, Re the Reynolds number, $W e$ the Weber number, and $F r$ the Froude number. Furthermore, the term $-\beta \hat{\mathbf{i}}$ is a vector with constant value $\beta$, which is responsible for the mass flow. In turn, the original pressure is replaced by the counterpart periodic $P$ through a decomposition [12], thus rendering the unknown to be found. Finally, the force field $\mathbf{f}$ amounts to the jump conditions existing on the interface in accordance with the "one-fluid" formulation and $\sigma$ is the surface tension of the liquid assumed as constant.

\section{Numerical Results}

Analyses of full 3D direct numerical simulations (DNS) performed for each model of array are displayed in this section for an air-water duo. To single out the ability of the numerical method to track the interfacial region of the bubbles, Fig. 2 depicts the computational representation of the periodic array $\mathcal{A}_{1}$ through a marker function separating the internal gas phase from the external liquid phase. 


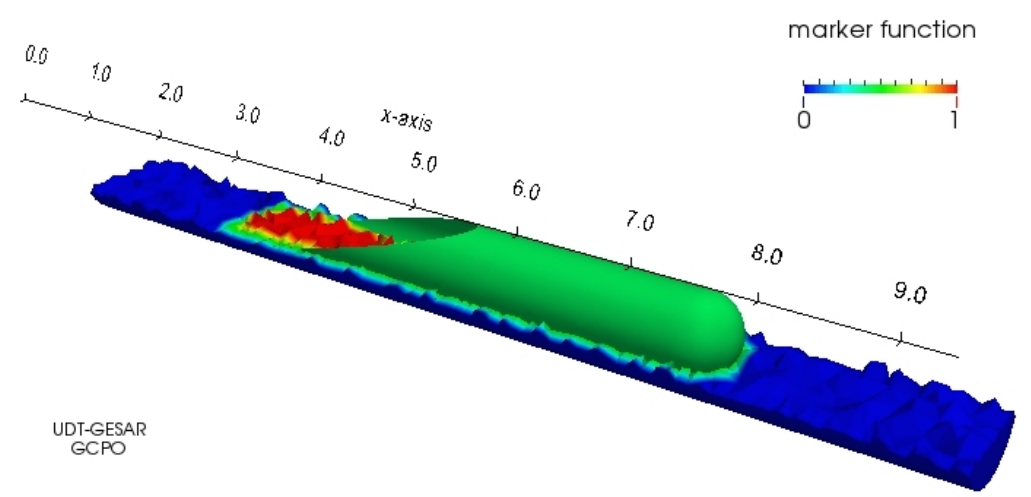

Figure 2: Overview of the gas-liquid periodic array for a single bubble highlighted by a marker function.

As can be seen through the inclined cut plane on the bubble body, inner and outer element layers meet at the bubble body so as to assure the propriety of thin thickness for the interface.

$(a)$

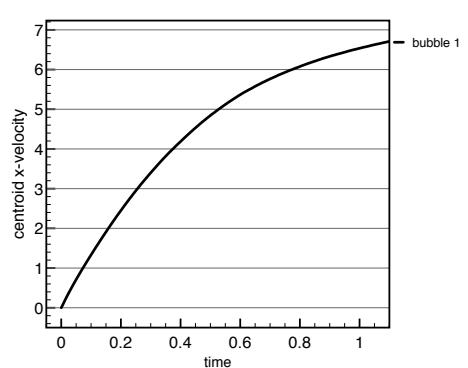

$(b)$

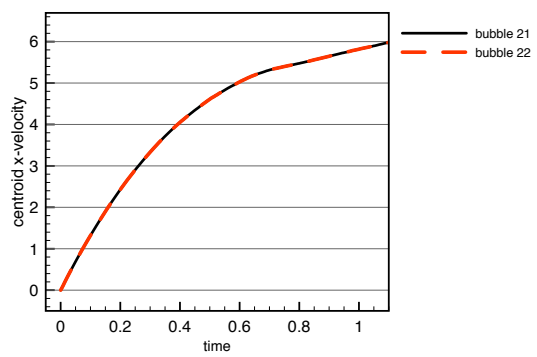

$(c)$

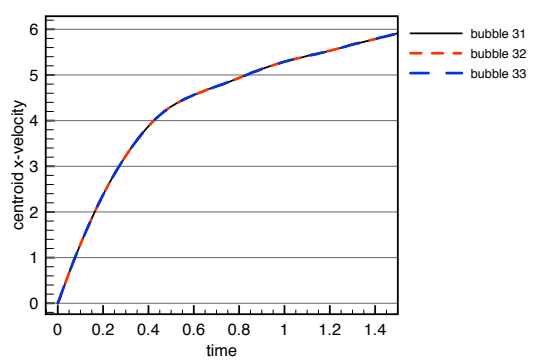

Figure 3: Bubbles' centroidal x-velocity profiles for the periodic arrays. $(a)$ array $1 ;(b)$ array $2 ;(c)$ array 3 .

Figure 3 depicts the profiles of the bubbles' centroidal $x$-velocity versus the dimensionless time for each setup of array, whereas Fig. 4 plots the elementaveraged mass flows calculated in the periodic boundaries in each case. From these figures, it can be inferred that breaking the longest bubble in two or three smaller bubbles reduces both their average velocity and respective average flow rates for the same value of the pressure gradient, thus supporting that these flow properties depend slightly on the array configuration.

Although the velocity profiles develop monotonically similar in each case, the acceleration experienced by the longest bubble in the array $\mathcal{A}_{1}$ is higher than those achieved in the other configurations. Furthermore, as depicted in Fig. 5, the overall behaviour of the velocity for both the bubbles of $\mathcal{A}_{2}$ and $\mathcal{A}_{3}$ is an identical motion within an equivalent simulation time.

Qualitative images of the pressure field of the periodic flows at a time step far from the initial condition are organized in Figs. (5-7).



Figure 4: Element-average flow rates for the periodic arrays obtained by weighting of the mean velocity on the periodic boundaries.

Since the pressure gradient affects the flow from left to right, higher pressures are felt by the trailing bubble of $\mathcal{A}_{2}$ and the outermost bubble of the train in $\mathcal{A}_{3}$. Equally verified are the high pressure regions around the trailing bubbles' caps due to a cumulative effect of wake interactions coming from the leading bubbles. In the liquid portion, emphasis was given to the mesh construction only, since the velocity field showed in the simulations was well uniform. By defining subscripts $a, w$ to stand for air, water, respectively, the physical properties set for the computations are specified in Table 1 , from which $R e \approx 14.58$, $W e \approx 0.20$, and $F r \approx 13,22$ are obtained. 


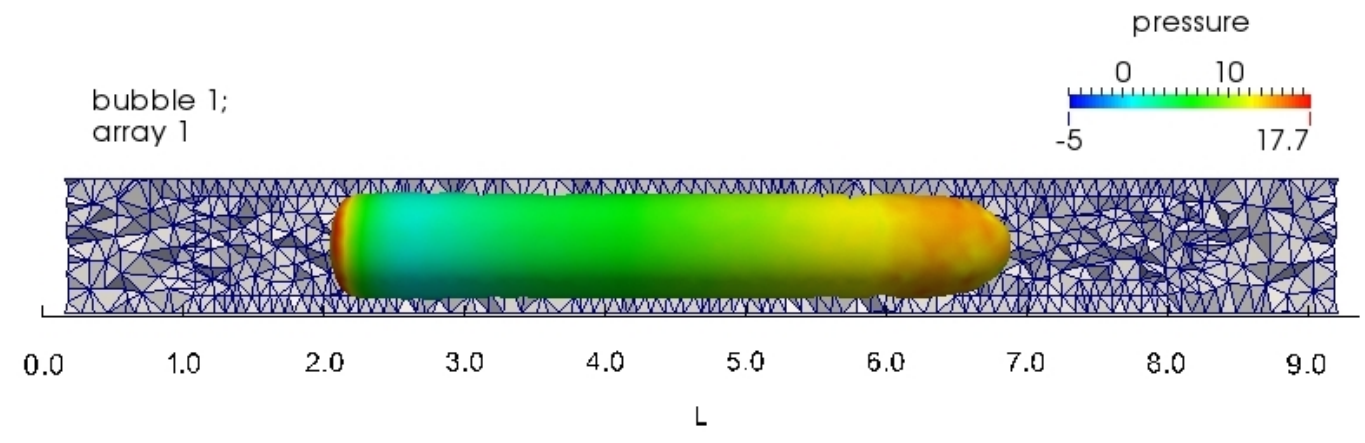

Figure 5: Pressure fields on the bubble's interface for the array 1 (single bubble).

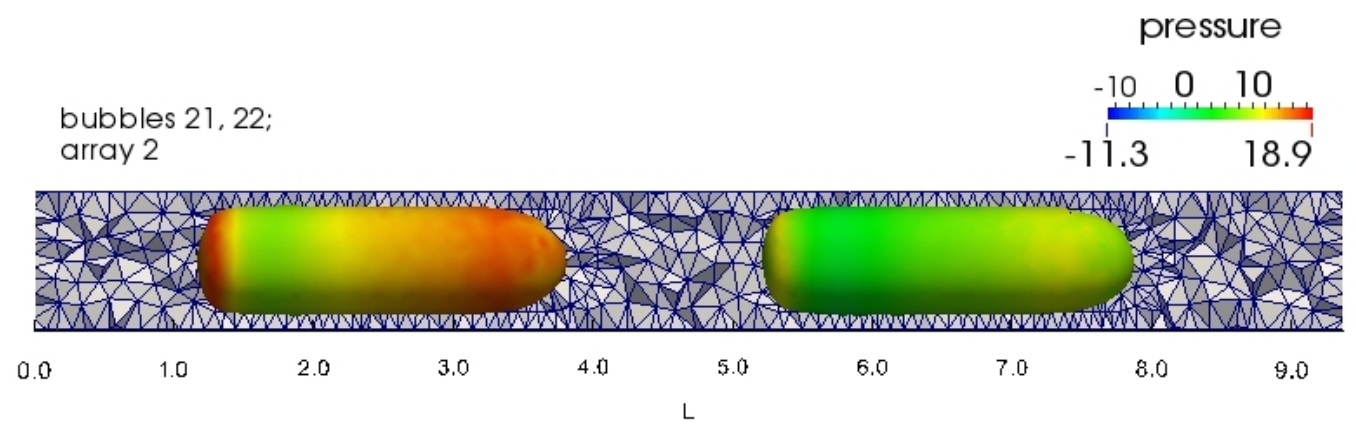

Figure 6: Pressure fields on the bubbles' interface for the array 2 (leading and sequential bubble).

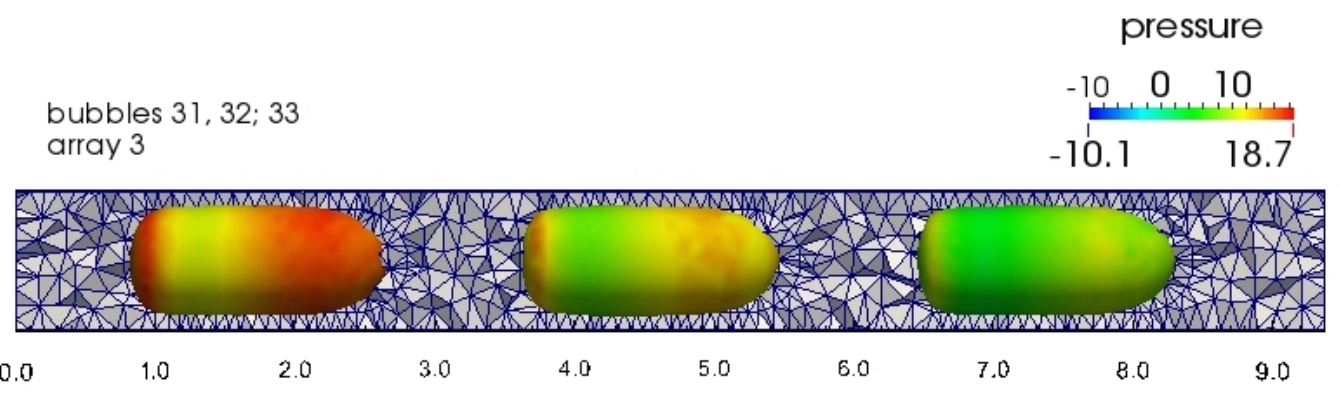

Figure 7: Pressure fields on the bubbles' interface for the array 3 (train of three bubbles).

Table 1: Physical properties used in the air-water simulation setups (in S.I. units).

\begin{tabular}{l|cc} 
Physical property & a & w \\
\hline \hline density $(\rho)$ & 1.205 & 998.63 \\
viscosity $(\mu)$ & $1.820 \times 10^{-5}$ & $1.002 \times 10^{-3}$ \\
surface tension $(\sigma)$ & - & 0.0728
\end{tabular}

\section{Conclusion}

This paper was intended to analyze comparatively the gas transport in microchannel slug flows through periodic domains. The effect of the imposition of a pressure gradient along with a moving frame technique allowed the establishment of economic simulation setups to assess the overall phenomena taking place inside a confined microchannel. Average quan- tities of the bubble velocity profiles and mass flow rates gave an insight about the transport of the fractioned gas and it was verified that the change of such quantities are slightly dependent on the array configuration.

By survey of the high pressure zones, we concluded that the trailing bubbles undergo cumulative influence both in the rear and cap regions, due to the forcing of the pressure gradient and the wake interac- 
tion of the front bubbles. Since the flow parameters characterized a laminar regime, considerable bubble asymmetries were not observed.

For future study, the inclusion of heat transfer sources as well as more accurate analyses of the gas fractioning through bubble trains is suggested, mainly for different pairs of substances, such as refrigerant fluids or even more viscous liquids.

\section{Acknowledgments}

G.P.O and N.M thank to the following Brazilian agencies for the sponsorship of this research: $\mathrm{CNPq} /$ Science Without Borders Program, CAPES and FAPERJ. J.P. acknowledges a fellowship from FAPERJ.

\section{References}

[1] Davies, RM, Taylor, G, 1950. The mechanics of large bubbles rising through extended liquids and through liquids in tubes, Proceedings of the Royal Society of London. Series A. Mathematical and Physical Sciences 200(1062): 375-390.

[2] Griffith, P, Wallis, GB, 1961. Two-phase slug flow, Journal of Heat Transfer 83(3): 307-318.

[3] Fabre, J, Lin, A, 1992. Modeling of two-phase slug flow, Annual Review of Fluid Mechanics 24(1): 21-46.

[4] Taha, T, Cui, ZF, 2004. Hydrodynamics of slug flow inside capillaries, Chemical Engineering Science 59(6): 1181-1190.

[5] Kashid, MN, Agar, DW, Turek, S, 2007. CFD modelling of mass transfer with and without chemical reaction in the liquidliquid slug flow microreactor, Chemical Engineering Science 62(18): 5102-5109.
[6] Cheng, L., Ribatski, G, Thome, JR, 2008. Twophase flow patterns and flow-pattern maps: fundamentals and applications, Applied Mechanics Reviews 61(5): 050802.

[7] Gupta, R., Fletcher, DF, Haynes, BS, 2009. On the CFD modelling of Taylor flow in microchannels, Chemical Engineering Science 64(12): 2941-2950.

[8] Chen, Y, Kulenovic, R, Mertz, R 2009. Numerical study on the formation of Taylor bubbles in capillary tubes, International Journal of Thermal Sciences 48(2): 234-242.

[9] Talimi, V, Muzychka, YS, Kocabiyik, S 2012. A review on numerical studies of slug flow hydrodynamics and heat transfer in microtubes and microchannels, International Journal of Multiphase Flow 39: 88-104.

[10] Magnini, M, Pulvirenti, B, Thome, JR 2013. Numerical investigation of the influence of leading and sequential bubbles on slug flow boiling within a microchannel, International Journal of Thermal Sciences 71:36-52.

[11] Anjos, GR, Borhani, N, Mangiavacchi, N, Thome, JR 2014. A 3D moving mesh Finite Element Method for two-phase flows, Journal of Computational Physics 270: 366-377.

[12] Mangiavacchi, N, Oliveira, GCP., Anjos, G, Thome, JR 2013. Numerical simulation of a periodic array of bubbles in a channel, Mecánica Computacional XXXII (21):1813-1824.

[13] Donea, J, Huerta, A, Ponthot, J-Ph, RodríguezFerran, A 2004. Arbitrary Lagrangian-Eulerian methods, Encyclopedia of computational mechanics, John Wiley \& Sons. 